\title{
Successful application of intra-arterial chemotherapy for advanced lacrimal sac carcinoma: a report of two cases
}

\author{
Remi Hibiya $^{1}$, Shinichi Ohba ${ }^{* 1}$, Mitsuhisa Fujimaki ${ }^{1}$, Masataka Kojima ${ }^{1}$, Junkichi Yokoyama $^{2}$ and Katsuhisa Ikeda ${ }^{1}$ \\ ${ }^{1}$ Department of Otorhinolaryngology-Head and Neck Surgery, Juntendo University Faculty of Medicine, Tokyo, Japan \\ ${ }^{2}$ Department of Otorhinolaryngology-Head and Neck Surgery, Moriyama Memorial Hospital, Nishikasai, Tokyo, Japan
}

\begin{abstract}
Objectives: Malignant lacrimal sac tumors are rare. The therapeutic methods are decided depending on the tumor status and general condition. A multidisciplinary approach may be needed. When necessary, orbital exenteration may be performed. To maintain the quality of life in patients, we applied intra-arterial infusion chemotherapy for locally advanced lacrimal sac squamous cell carcinoma cases.

Patients and methods: Two patients with lacrimal sac squamous cell carcinoma who were treated by intra-arterial chemotherapy were included in this study. Intraarterial chemotherapy was administered at $150 \mathrm{mg} / \mathrm{m}^{2}$ of CDDP and $15 \mathrm{mg} / \mathrm{m}^{2}$ of DTX. Radiotherapy in combination with intra-arterial chemotherapy was performed for one patient. A Cyberknife was used for the other patient.

Result: In both cases, the tumor was considered to have disappeared judging from the PET scan. Furthermore, the eyes of these patients were spared and the visual function was preserved.

Conclusion: Multidisciplinary management should be applied for malignant lacrimal sac tumor. Intra-arterial chemotherapy can play an important role in organ preservation therapy for locally advanced lacrimal sac squamous cell carcinoma.
\end{abstract}

\section{Introduction}

Malignant lacrimal sac tumors are rare. The most common malignant tumors are of epithelial origin, with squamous cell carcinoma. The management of malignant tumors includes local resection or extensive wide resection with orbital exenteration followed by adjuvant therapy. However, orbital exenteration decreases the quality of life in such patients.

This is the first report describing the application of superselective intra-arterial chemotherapy for locally advanced lacrimal sac carcinoma. This procedure could be a useful treatment for functional preservation of the orbit.

\section{Patients and methods}

Two patients with locally advanced lacrimal sac squamous cell carcinoma were treated with superselective intra-arterial chemotherapy. In these patients, $150 \mathrm{mg} / \mathrm{m}^{2}$ of CDDP and $15 \mathrm{mg} / \mathrm{m}^{2}$ of DOC were superselectively administered weekly to each feeding artery including the internal carotid artery. CT angiography was performed to confirm that the entire tumor was stained by contrast agents via the femoral approach. The one patient was treated with concomitant chemo-radiation and the other subsequently underwent Cyber knife treatment.

\section{Results}

\section{Case 1}

A 72 year-old female presented with right exophthalmous and diplopia. She had been treated for dacryocystitis for two years. On clinical examination, she was noted to have a firm mass inferior to the right medial canthas. The tumor was also easily visible in the right inferior nasal meatus (Figure1A). CT of the sinuses revealed an enhanced mass in the right lacrimal sac extensively involving the right orbit. There was also extension of the mass into the nasal cavity, the ethomoid sinus (Figure1B). Transnasal biopsy revealed moderately differentiated squamous cell carcinoma.

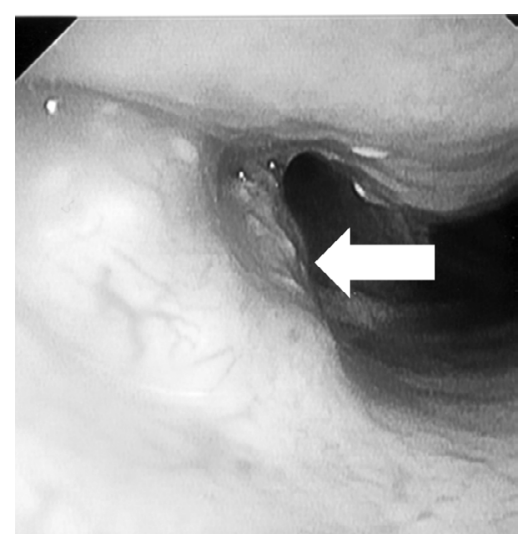

Figure 1A. A tumor was confirmed in the right inferior nasal meatus (arrows). Trans nasal biopsy was performed, which revealed moderately differentiated squamous cell carcinoma.

Correspondence to: Dr. Shinichi Ohba, Department of OtorhinolaryngologyHead and Neck Surgery, Juntendo University Faculty of Medicine, Tokyo, Japan; Tel:+ 81-3-3813-3111; Fax: 81-3-5840-7103; E-mail: sooba@juntendo.ac.jp

Received: September 30, 2016; Accepted: October 31, 2016; Published: November 03, 2016 

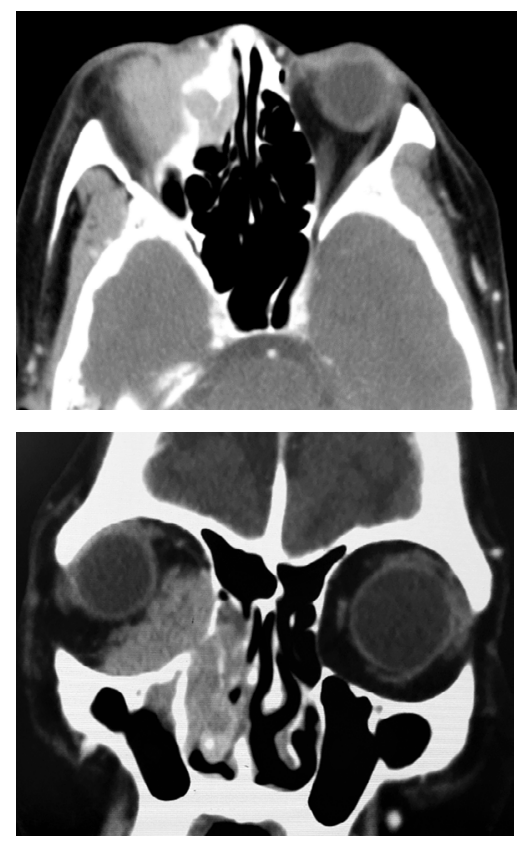

Figure 1B. Appearance of tumor on CT scan: axial CT scan shows enhancement of this lesion extending to the right orbit; coronal CT revealing the enhanced mass widely involving the orbit with bone destruction.

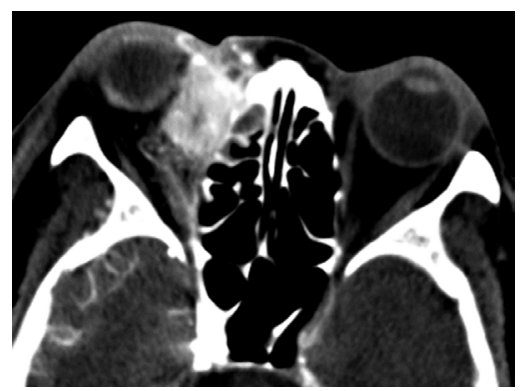

Figure 2. CT angiographies of the right internal carotid artery. The cancer area that was not stained by the maxillary artery was covered by infusion of the right internal carotid artery.

To avoid orbital exenteration, intra-arterial chemotherapy with concurrent radiotherapy was conducted five times. All of the cancer area was enhanced by contrast agent in the CT angiography, not only the external carotid artery but also the internal carotid artery (Figure 2). $200 \mathrm{mg}$ of cisplatin and $20 \mathrm{mg}$ of docetaxel were administered weekly five times. During the infusion of CDDP, sodium thiosulphate (STS) at 200 fold the dose of CDDP was injected to the brachiocephalic vein to neutralize the CDDP. The procedure was performed weekly for five times. The primary tumor was irradiated for a total $60 \mathrm{~Gy}$ in 30 fractions.

Two months after the treatment, MRI and 18F-FDG PET/CT showed no evidence of residual disease (Figure $3 \mathrm{~A}$ and $3 \mathrm{~B}$ ). There were no complications related to the ICA infusion. The visual acuity was unchanged after treatment and the diplopia was also improved.

\section{Case 2}

A 68-year-old-man was referred to our hospital with a hard mass in theleft medial canthus and tearing. Hehad been diagnosed with maxillary sinus cancer at the age of 40 years and received chemoradiotherapy followed by partial maxillectomy at that time. He had also undergone coronary artery bypass surgery. Physical examination revealed a firm and fixed mass in the left internal canthus. An otolaryngologic rigid nasal/sinus endoscopy showed a firm mass under the normalappearing mucosa just in front of the uncinate process (Figure 4A). Transnasal biopsy revealed moderately differentiated squamous cell carcinoma that was considered to be a radiation-induced cancer. A sinus CT demonstrated a massively enlarged left lacrimal sac with bone loss. There was also extension of the mass into the orbit (Figure 4B). The patient's visual acuity was 20/20 and 20/20.

The patient was treated with super-selective intra-arterial chemotherapy followed by Cyberknife therapy (an-image guided, robotic radiotherapy) as an organ preservation technique. Most of the tumor was fed by the internal carotid artery (ICA) (Figure 5). $125 \mathrm{mg}$ of CDDP and $10 \mathrm{mg}$ of DOC were administered weekly four times (day 1,8,15 and 22) via the superficial temporal artery. The cyberknife therapy ( 3 treatments; maximum dose, $3703 \mathrm{cGy}$ ) was performed a month after the chemotherapy. The post treatment PET scan taken after 2 months revealed there was no residual tumor (Figure 6). There was no adverse event related to the ICA infusion and the visual acuity was preserved at $20 / 20$ and $20 / 20$.

\section{Discussion}

A variety of neoplasms may affect the lacrimal drainage system, with approximately $70 \%$ malignant in nature [1]. The most common type of cancer is squamous cell carcinoma followed by transitional cell carcinoma [2,3]. Lacrimal sac carcinoma is often mistaken for
A

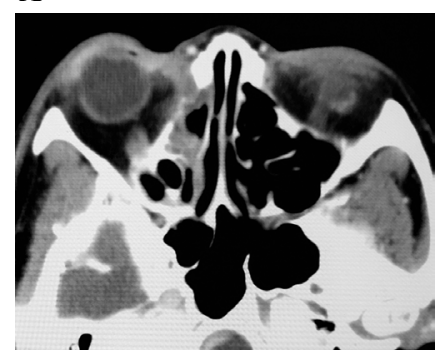

B

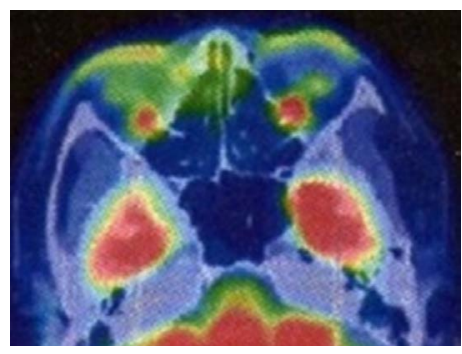

Figure 3. CT scan [Figure 3A] and FDG-PET/CT [Figure 3B] at two months after treatment showing no significant accumulation in the right lacrimal sac.
A

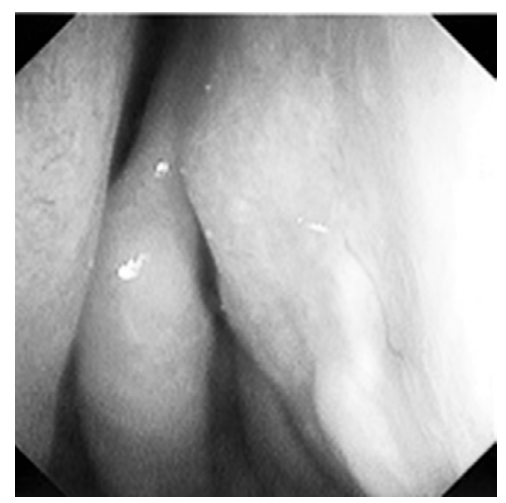

B

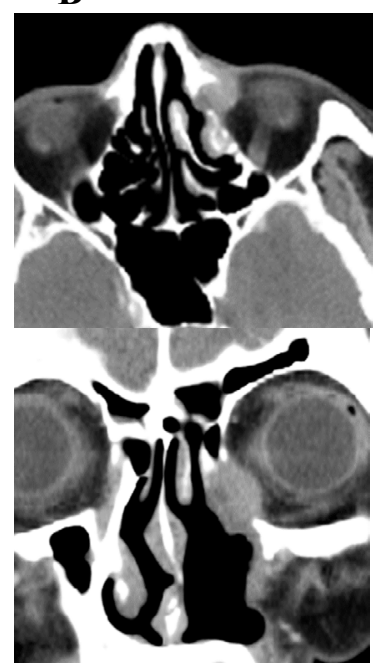

Figure 4. A firm mass under the normal-appearing mucosa just in front of the uncinate process [Figure 4A] CT scan showing an enhanced enlarged left lacrimal sac with bone loss. The enhanced mass extended into the orbit [Figure 4B]. 


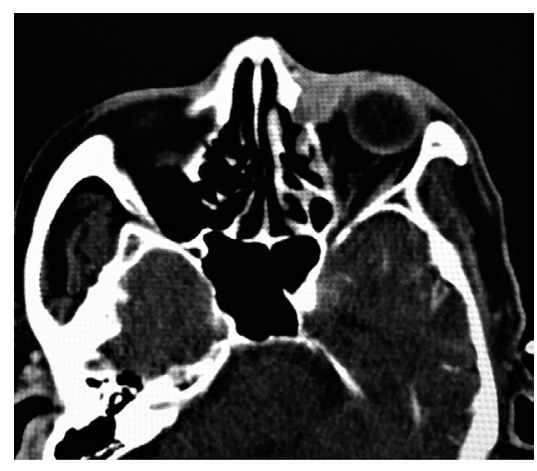

Figure 5. CT angiography of the left internal carotid artery. The tumor staining was confirmed.

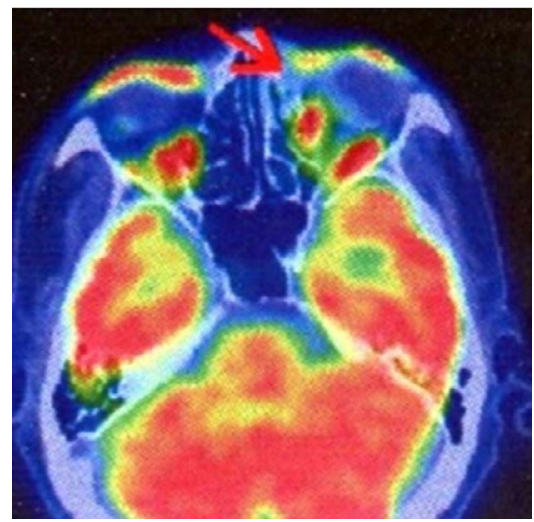

Figure 6. FDG-PET/CT of the post treatment. The absence of a tumor was confirmed.

dacryocystitis due to the similar symptomatology [3-5]. As a result, the diagnosis and the definitive treatment are often delayed as in our two cases.

The management of primary lacrimal sac malignancies is complex and varies from case to case [1]. The treatment of such tumors is first and foremost complete surgical removal [5]. Local resection may be indicated for localized lesions, but, wide resection with exenteration is needed for advanced disease.

Chemoradiotherapy has significantly enabled the preservation of important organs in the treatment of head and neck cancer. Since resistance to systemic chemotherapy has been reported especially in advanced sinonasal cancer cases [6,7], intra-arterial chemotherapy, which increases the concentration of anticancer drug in the tumor, has become a feasible alternative method [8-11]. Conventionally, anticancer drugs are administered only to the branches of the external carotid artery to preventadverse events from infusion of the internal carotid artery. However, Yokoyama J, et al. has reported the feasibility and safety of intra-arterial chemotherapy including the internal carotid artery for advanced sinus cancers involving the skull base [11]. In that report, this new method of the intra-arterial chemotherapy could contribute to the preservation of the orbial contents and visual function [11].

Orbital exenteration should be considered if the tumor invades to the orbital cone or when there is tumor recurrence after radiotherapy [5]. This leads to devastating cosmetic, functional and psychological problems in patients even when reconstructive surgery or orbital prosthesis is considered. Furthermore, $\mathrm{Ni} \mathrm{C}$, et al. showed that, when exenteration is required, the prognosis remains poor, with mortality reaching $50 \%$ even in cases treated by radical surgery with tumor excision and orbit exenteration followed by radiation treatment [12]. Also Suarez, et al. reported that deep involvement of the orbit in sinonasal tumors not only affected the preservation of the orbital contents but was also a significant factor for poor survival [13]. Considering minimally invasive treatment with organ preservation in advanced lacrimal sac cancer, intra-arterial chemotherapy including infusion of the internal carotid artery may be a useful and safe method. The side effects related to intra-arterial infusion to the ICA have been acceptable in our experience of two cases. However, tumor recurrence or delayed toxicity related to the radiation therapy such as xerophthalmia, radiation retinopathy, glaucoma, cataract or keratitis with corneal uncerations should be monitored carefully.

In conclusion, intra-areterial chemotherapy may become a feasible treatment for advanced lacrimal sac cancer even for tumors fed by the internal carotid artery.

\section{References}

1. Harrison LB, Sessions RB, Kies MS (2014) Site-specific principles of management of head and neck cancer. 4th edition. Section2.Wolters Kluwer Health. 544-545.

2. Stefanyszyn MA, Hidayat AA, Pe'er JJ, Flanagan JC (1994) Lacrimal sac tumors Ophthal Plast Reconstr Surg 10: 169-184. [Crossref]

3. El-Sawy T, Frank SJ, Hanna E, Sniegowski M, Lai SY, et al. (2013) Multidisciplinary management of lacrimal sac/nasolacrimal duct carcinomas. Ophthal Plast Reconstr Surg 29: 454-457. [Crossref]

4. Katircioglu YA, Altiparmak UE, Akmansu H, Ustün H, Zorlu F, et al. (2003) Squamous cell carcinoma of the lacrimal sac. Orbit 22: 151-153. [Crossref]

5. Montalban A, Liétin B, Louvrier C, Russier M, Kemeny JL, et al. (2010) Malignant lacrimal sac tumors. Eur Ann Otorhinolaryngol Head Neck Dis 127: 165-172. [Crossref]

6. Munro AJ (1995) An overview of randomised controlled trials of adjuvant chemotherapy in head and neck cancer. Br J Cancer 71: 83-91. [Crossref]

7. El-Sayed S, Nelson N (1996) Adjuvant and adjunctive chemotherapy in the management of squamous cell carcinoma of the head and neck region. A meta-analysis of prospective and randomized trials. J Clin Oncol 14: 838-847. [Crossref]

8. Tomura N, Hashimoto M, Sashi R, Hirano H, Kobayashi M, et al. (1996) Superselective angio-CT of brain tumors. AJNR Am J Neuroradiol 17: 1073-1080. [Crossref]

9. Hirai T, Korogi Y, Ono K, Maruoka K, Harada K, et al. (2001) Intraarterial chemotherapy or chemoembolization for locally advanced and/or recurrent hepatic tumors: evaluation of the feeding artery with an interventional CT system. Cardiovasc Intervent Radiol 24: 176-179. [Crossref]

10. Miyayama S, Yamashiro M, Hattori Y, Orito N, Matsui K, et al. (2011) Usefulness of $\mathrm{C}$-arm CT during superselective infusion chemotherapy for advanced head and neck carcinoma. J Med Imaging Radiat Oncol 55: 368-372. [Crossref]

11. Yokoyama J, Ohba S, Fujimaki M, Anzai T, Kojima M, et al. (2014) Impact of intraarterial chemotherapy including internal carotid artery for advanced paranasal sinus cancers involving the skull base. Br J Cancer. 111: 2229-2234. [Crossref]

12. Ni C, D'Amico DJ, Fan CQ, Kuo PK (1982) Tumors of the lacrimal sac: a clinicopathological analysis of 82 cases. Int Ophthalmol Clin 22: 121-140. [Crossref]

13. Suarez C, Llorente JL, Fernandez De Leon R, Maseda E, Lopez A (2004) Prognostic factors in sinonasal tumors involving the anterior skull base. Head Neck 26: 136-144. [Crossref]

Copyright: $(2016$ Hibiya R. This is an open-access article distributed under the terms of the Creative Commons Attribution License, which permits unrestricted use, distribution, and reproduction in any medium, provided the original author and source are credited. 